\title{
A Note on Isomorphism Theorems for Semigroups of Order-Preserving Transformations with Restricted Range
}

\author{
Phichet Jitjankarn $^{1}$ and Thitarie Rungratgasame ${ }^{2}$ \\ ${ }^{1}$ Division of Mathematics, School of Science, Walailak University, Nakhon Si Thammarat 80161, Thailand \\ ${ }^{2}$ Department of Mathematics, Faculty of Science, Srinakharinwirot University, Bangkok 10110, Thailand \\ Correspondence should be addressed to Phichet Jitjankarn; jitjankarn@gmail.com
}

Received 13 October 2014; Accepted 2 February 2015

Academic Editor: David E. Dobbs

Copyright (c) 2015 P. Jitjankarn and T. Rungratgasame. This is an open access article distributed under the Creative Commons Attribution License, which permits unrestricted use, distribution, and reproduction in any medium, provided the original work is properly cited.

\begin{abstract}
Finding necessary and sufficient conditions for isomorphism between two semigroups of order-preserving transformations over an infinite domain with restricted range was an open problem. In this paper, we show a proof strategy to answer that question.
\end{abstract}

\section{Introduction}

For a nonempty set $X$, let $T(X)$ be the full transformation semigroup under composition of all maps from $X$ to $X$. When $X$ is a partially ordered set (poset), a mapping $\alpha$ in $T(X)$ is called order-preserving if $x \leq y$ implies $x \alpha \leq y \alpha$ for all $x, y \in X$, and $\alpha$ is regressive if $x \alpha \leq x$ for all $x \in X$. We denote by $T_{\mathrm{OP}}(X)$ and $T_{\mathrm{RE}}(X)$ the subsemigroups of $T(X)$ of all order-preserving maps and all regressive maps on $X$, respectively. The semigroups of order-preserving maps were first introduced by Howie in [1].

For $\alpha \in T(X)$, let $\operatorname{ran} \alpha$ denote the range of $\alpha$. In 1975 , Symons [2] introduced and studied the subsemigroup $T\left(X, X^{\prime}\right)$ where $\emptyset \neq X^{\prime} \subseteq X$ of $T(X)$ consisting of $\alpha \in T(X)$ with $\operatorname{ran} \alpha \subseteq X^{\prime}$. Subsemigroups of transformations (with restricted range) of $T(X)$ of this type have been studied extensively, including our work which we will mention later on. Regarding the semigroups of regressive type, in 1996, Umar proved that for any chains $X$ and $Y, T_{\mathrm{RE}}(X) \cong T_{\mathrm{RE}}(Y)$ if and only if $X$ and $Y$ are order-isomorphic (see in [3]). Later in [4], Saitô et al. generalized this result to partially ordered sets. They introduced the adjusted partially ordered set $A(X)$ of a poset $X$ and proved that the order-isomorphism between $A(X)$ and $A(Y)$ is a necessary and sufficient condition for the two semigroups to be isomorphic.

In this paper, we are also interested in studying the isomorphisms of subsemigroups of transformations with restricted range. Now, let us introduce the subsemigroups which will be of particular interest to us in this paper.

For a partially ordered set $X$ and a subset $X^{\prime}$ of $X$, we let

$$
\begin{aligned}
& T_{\mathrm{OP}}\left(X, X^{\prime}\right):=T_{\mathrm{OP}}(X) \cap T\left(X, X^{\prime}\right), \\
& T_{\mathrm{RE}}\left(X, X^{\prime}\right):=T_{\mathrm{RE}}(X) \cap T\left(X, X^{\prime}\right) .
\end{aligned}
$$

Then both of these are subsemigroups of $T\left(X, X^{\prime}\right)$.

In 2012, Udomkavanich and Jitjankarn proved in [5] that $T_{\mathrm{RE}}\left(X, X^{\prime}\right) \cong T_{\mathrm{RE}}\left(Y, Y^{\prime}\right)$ if and only if two adjusted chains $\mathscr{A}\left(X, X^{\prime}\right)$ and $\mathscr{A}\left(Y, Y^{\prime}\right)$ are order-structural isomorphic. This result leads us to study the isomorphism theorems for the semigroups of order-preserving type. It is known (e.g., [6], pages 222-223) that, for posets $X$ and $Y, T_{\mathrm{OP}}(X) \cong T_{\mathrm{OP}}(Y)$ if and only if $X$ and $Y$ are either order-isomorphic or orderanti-isomorphic. These necessary and sufficient conditions also hold for the isomorphisms on the semigroups of partial order-preserving transformations (see in [7]). In 2014, Fernandes et al. [8] show that these conditions apply for $T_{\mathrm{OP}}\left(X, X^{\prime}\right)$ and $T_{\mathrm{OP}}\left(Y, Y^{\prime}\right)$ to be isomorphic when $X$ and $Y$ are finite as well. In this paper, we study the case when $X$ and $Y$ are infinite chains. Since $T_{\mathrm{OP}}\left(X, X^{\prime}\right)$ is trivial when $\left|X^{\prime}\right|=1$, we omit this case.

Throughout the paper, we assume that $X$ and $Y$ are chains, $\left|X^{\prime}\right|>1$, and $\left|Y^{\prime}\right|>1$. The following statement is known. 

that

If there is an order-(anti)-isomorphism $\theta: X \rightarrow Y$ such

$$
\left(X^{\prime}\right) \theta=Y^{\prime} \quad \text { for some } X^{\prime} \subseteq X, Y^{\prime} \subseteq Y \text {, }
$$

then $T_{\mathrm{OP}}\left(X, X^{\prime}\right) \cong T_{\mathrm{OP}}\left(Y, Y^{\prime}\right)$.

It is natural to ask whether the converse of the above result holds. Nevertheless, our work shows that it may not be the case if $\left|X^{\prime}\right|=2$. To be precise, we derive that the converse of the statement (2) holds when $\left|X^{\prime}\right| \geq 3$.

To prove the statements, we apply in a similar fashion to [5] the idea of using adjusted chains. To do so, we will first introduce some notation and definitions that will be useful in Section 2. In Section 3, some homomorphism properties which are preserved under isomorphism will be given. Lastly, the isomorphism theorems for the semigroups of the type $T_{\mathrm{OP}}\left(X, X^{\prime}\right)$ when $X$ is an infinite chain are determined in Section 4 .

\section{Basic Notations and Results}

Let $\mathscr{C}^{\prime}$ be a subchain of a chain $\mathscr{C}$. Let $\left\{\mathscr{C} \backslash \mathscr{C}^{\prime}\right\}$ denote the set of all equivalence classes of $\mathscr{C} \backslash \mathscr{C}^{\prime}$ such that each class contains all elements in $\mathscr{C} \backslash \mathscr{C}^{\prime}$ with no elements in $\mathscr{C}^{\prime}$ lying between them. Then we consider $\left\{\mathscr{C} \backslash \mathscr{C}^{\prime}\right\} \cup \mathscr{C}^{\prime}$ as a chain under the partial order induced by the chain $\mathscr{C}$ in the natural way. This chain is an adjusted chain, denoted by $\mathscr{A}\left\{\mathscr{C}, \mathscr{C}^{\prime}\right\}$.

For each $a, b \in \mathscr{C}$ with $a<b$, the intervals $(a, b),[a, b)$, $(a, b],[a, b]$ in $\mathscr{C}$ are defined naturally and we define the following intervals:

$$
\begin{array}{ll}
(\leftarrow a]:=\{z \in \mathscr{C}: z \leq a\}, \quad[a \rightarrow):=\{z \in \mathscr{C}: z \geq a\}, \\
(\leftarrow a):=\{z \in \mathscr{C}: z<a\}, \quad(a \rightarrow):=\{z \in \mathscr{C}: z>a\} .
\end{array}
$$

For a nonempty subset $V$ of a chain $\mathscr{C}, V$ is said to be convex if for $x, y, z \in \mathscr{C}$ such that $x \leq z \leq y, x, y \in V$ implies $z \in V ; V$ is called an upper(lower)-convex subset of $\mathscr{C}$ if $x<y$ $(x>y)$ for all $x \in \mathscr{C} \backslash V$ and $y \in V$.

For a convex subset $V$ of $\mathscr{C}$, we define

$$
(\leftarrow V):=\{z \in \mathscr{C} \backslash V: z \text { is a lower bound of } V \text { in } \mathscr{C}\} \text {, }
$$$$
(V \rightarrow):=\{z \in \mathscr{C} \backslash V: z \text { is an upper bound of } V \text { in } \mathscr{C}\} \text {. }
$$

For convenience, if $a \in \mathscr{C}$, let $\mathscr{C}_{a}$ be the element of $T(\mathscr{C})$ whose range is $\{a\}$.

Given $[k] \in\left\{\mathscr{C} \backslash \mathscr{C}^{\prime}\right\}, k \in \mathscr{C} \backslash \mathscr{C}^{\prime}$. We will define some order-preserving maps of $T\left(\mathscr{C}, \mathscr{C}^{\prime}\right)$ as follows.

(i) For a convex subset $A$ of $[k]$ and $a, b, c \in \mathscr{C}^{\prime}$ such that $a \leq b<[k]<c($ or $a<[k]<b \leq c)$, we write

$$
\omega_{a: A_{b}: c}:=\left(\begin{array}{ccc}
(\leftarrow A) & A & (A \rightarrow) \\
a & b & c
\end{array}\right),
$$

where $\operatorname{ran}\left(\omega_{a: A_{b}: c}\right)=\{a, b, c\}$ and $b\left(\omega_{a: A_{b}: c}\right)^{-1}=A$ if $a \neq b$.

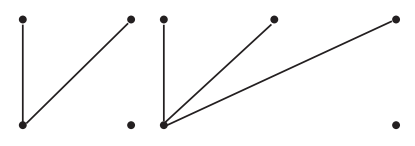

FIGURE 1

(ii) When $[k]=\min \mathscr{A}\left\{X \backslash X^{\prime}\right\}$, for a lower-convex subset $L$ of $[k]$ and $a, b \in \mathscr{C}^{\prime}$ such that $[k]<a<b$, we write

$$
\omega_{L_{a}: b}:=\left(\begin{array}{cc}
L & (L \rightarrow) \\
a & b
\end{array}\right),
$$

where $\operatorname{ran}\left(\omega_{L_{a}: b}\right)=\{a, b\}$ and $a\left(\omega_{L_{a}: b}\right)^{-1}=L$.

(iii) When $[k]=\max \mathscr{A}\left\{X \backslash X^{\prime}\right\}$, for an upper-convex subset $U$ of $[k]$ and $a, b \in \mathscr{C}^{\prime}$ such that $a<b<[k]$, we write

$$
\omega_{a: U_{b}}:=\left(\begin{array}{cc}
(\leftarrow U) & U \\
a & b
\end{array}\right),
$$

where $\operatorname{ran}\left(\omega_{a: U_{b}}\right)=\{a, b\}$ and $b\left(\omega_{a: U_{b}}\right)^{-1}=U$.

For $\alpha \in T(\mathscr{C})$, we denote $\operatorname{Fix}(\alpha)=\{x \in \mathscr{C}: x \alpha=x\}$.

For $\alpha \in T\left(\mathscr{C}, \mathscr{C}^{\prime}\right)$, we define the partial graph of transformation $\alpha$, denoted by $\Gamma_{\alpha}:=\left(\mathscr{C}^{\prime}, \operatorname{ran} \alpha, E_{\alpha}\right)$, in the following way: $\mathscr{C}^{\prime}$ is the set of upper vertices, $\operatorname{ran} \alpha$ is the set of lower vertices such that all vertices are placed in order, and $E_{\alpha}$ is the set of (directed) edges which each element is in the form $x \alpha y$, where $x \alpha=y$ for $x, y \in \mathscr{C}^{\prime}$. Notice that the number of components in each partial graph is equal to the number of elements in its range. Furthermore, the components, considered from left to right, are placed in the same order as their related elements in the range.

Example 1. For the transformation $\alpha \in T(\{1,2, \ldots, 9\},\{1,3$, $5,7,9\}$ ) defined by

$$
\alpha=\left(\begin{array}{lllllllll}
1 & 2 & 3 & 4 & 5 & 6 & 7 & 8 & 9 \\
1 & 5 & 1 & 9 & 5 & 5 & 5 & 3 & 5
\end{array}\right),
$$

the set of upper vertices is $\{1,3,5,7,9\}$, the set of lower vertices is $\{1,3,5,9\}$, and $E_{\alpha}=\{1 \alpha 1,3 \alpha 1,5 \alpha 5,7 \alpha 5,9 \alpha 5\}$. Then the graph $\Gamma_{\alpha}$ has the form shown in Figure 1.

The partial graph $\Gamma_{\alpha}$ has four components placed in order from left to right.

Theorem 2. If $T_{\mathrm{OP}}\left(X, X^{\prime}\right) \cong T_{\mathrm{OP}}\left(Y, Y^{\prime}\right)$, then $X^{\prime}$ and $Y^{\prime}$ are either order-isomorphic or order-anti-isomorphic.

Proof. Let $\varphi: T_{\mathrm{OP}}\left(X, X^{\prime}\right) \rightarrow T_{\mathrm{OP}}\left(Y, Y^{\prime}\right)$ be an isomorphism. For each $a \in X^{\prime}$, there is an element $\bar{a} \in Y^{\prime}$ such that $\left(X_{a}\right) \varphi=$ $Y_{\bar{a}}$ by idempotent and right zero properties of $X_{a}$ and $Y_{\bar{a}}$. The map $a \mapsto \bar{a}$ becomes a bijective map from $X^{\prime}$ onto $Y^{\prime}$. It remains to show that this map is either order-preserving or order-anti-preserving. Let $a, b, s, t \in X^{\prime}$ be such that $a<b$ and $s<t$. Since $Y^{\prime}$ is a chain and the map is one-to-one, it 
must be that $\bar{a}<\bar{b}$ or $\bar{a}>\bar{b}$ and $\bar{s}<\bar{t}$ or $\bar{s}>\bar{t}$. Now, we have $(\underset{s}{(\leftarrow a]} \underset{t}{(a \rightarrow)}) \in T_{\mathrm{OP}}\left(X, X^{\prime}\right)$ such that

$$
\begin{aligned}
& X_{a}\left(\begin{array}{cc}
(\leftarrow a] & (a \rightarrow) \\
s & t
\end{array}\right)=X_{s}, \\
& X_{b}\left(\begin{array}{cc}
(\leftarrow a] & (a \rightarrow) \\
s & t
\end{array}\right)=X_{t} .
\end{aligned}
$$

Then

$$
\begin{aligned}
& Y_{\bar{a}}\left(\left(\begin{array}{cc}
(\leftarrow a] & (a \rightarrow) \\
s & t
\end{array}\right) \varphi\right)=Y_{\bar{s}} \\
& Y_{\bar{b}}\left(\left(\begin{array}{cc}
(\leftarrow a] & (a \rightarrow) \\
s & t
\end{array}\right) \varphi\right)=Y_{\bar{t} .}
\end{aligned}
$$

Consequently,

$$
\bar{a}\left(\begin{array}{cc}
(\leftarrow a] & (a \rightarrow) \\
s & t
\end{array}\right) \varphi=\bar{s}, \quad \bar{b}\left(\begin{array}{cc}
(\leftarrow a] & (a \rightarrow) \\
s & t
\end{array}\right) \varphi=\bar{t} .
$$

Since $(\underset{s}{(\leftarrow a](\underset{t}{(a \rightarrow})}) \varphi \in T_{\mathrm{OP}}\left(Y, Y^{\prime}\right)$, it follows that $\bar{a}<\bar{b}$ implies $\bar{s}<\bar{t}$ and $\bar{a}>\bar{b}$ implies $\bar{s}>\bar{t}$. This proves that $X^{\prime}$ and $Y^{\prime}$ are either order-isomorphic or order-anti-isomorphic.

From now on, let $\varphi$ denote an isomorphism from $T_{\mathrm{OP}}\left(X, X^{\prime}\right)$ and $T_{\mathrm{OP}}\left(Y, Y^{\prime}\right)$. The order-(anti)-isomorphism from $X^{\prime}$ onto $Y^{\prime}$, defined in the proof of Theorem 2, is denoted by $\theta_{\varphi}$. It is easy to see that the order-(anti)-isomorphism $\theta_{\varphi^{-1}}$ from $Y^{\prime}$ onto $X^{\prime}$, induced by the isomorphism $\varphi^{-1}$, is the inverse function of $\theta_{\varphi}$. That is,

$$
\theta_{\varphi^{-1}}=\left(\theta_{\varphi}\right)^{-1}
$$

Notice that, by considering $\varphi^{-1}$ and $\theta_{\varphi^{-1}}$ instead of $\varphi$ and $\theta_{\varphi}$, respectively, all results that hold for $\varphi$ also hold for $\varphi^{-1}$.

\section{Some Homomorphism Properties}

In this section, we study some properties of transformations which will be preserved under a homomorphism. First, we will study the structure of $\alpha$ and $\alpha \varphi$ through $\theta_{\varphi}$ when $\alpha \epsilon$ $T_{\mathrm{OP}}\left(X, X^{\prime}\right)$. Then we derive that two graphs of $\Gamma_{\alpha}$ and $\Gamma_{(\alpha) \varphi}$ are isomorphic. Moreover, the order of components (in the sense of partial graph) is also preserved.

Without loss of generality, we assume that $\theta_{\varphi}$ is orderpreserving from now on. The other case that $\theta_{\varphi}$ is order-antipreserving can be done by the same process.

Lemma 3. For each $\alpha \in T_{O P}\left(X, X^{\prime}\right)$, the following statements hold.

(i) $(\operatorname{Fix}(\alpha)) \theta_{\varphi}=\operatorname{Fix}(\alpha \varphi)$. (ii) For $a \in \operatorname{ran} \alpha$ such that $a \alpha^{-1} \cap X^{\prime} \neq \emptyset$,

$$
\bar{a} \in \operatorname{ran}(\alpha \varphi), \quad \bar{a}(\alpha \varphi)^{-1} \cap Y^{\prime}=\left(a \alpha^{-1} \cap X^{\prime}\right) \theta_{\varphi} .
$$

In particular, if $\alpha$ is an idempotent, then $(\operatorname{ran} \alpha) \theta_{\varphi}=\operatorname{ran}(\alpha \varphi)$.

Proof. (i) Let $a \in \operatorname{Fix}(\alpha)$. Then $a \alpha=a$. Since $Y_{\bar{a}}(\alpha \varphi)=$ $\left(X_{a} \varphi\right)(\alpha \varphi)=\left(X_{a} \alpha\right) \varphi=X_{a} \varphi=Y_{\bar{a}}$, it follows that $\bar{a}(\alpha \varphi)=$ $\bar{a}=a \theta_{\varphi} \in \operatorname{Fix}(\alpha \varphi)$. Similarly, if $\bar{s} \in \operatorname{Fix}(\alpha \varphi)$, then $X_{s} \alpha=$ $\left(Y_{\bar{s}} \varphi^{-1}\right) \alpha=\left(Y_{\bar{s}}(\alpha \varphi)\right) \varphi^{-1}=\left(Y_{\bar{s}}\right) \varphi^{-1}=X_{s}$; that is, $s \alpha=s$. Then $\bar{s}=s \theta_{\varphi} \in(\operatorname{Fix}(\alpha)) \theta_{\varphi}$.

(ii) For $a \in \operatorname{ran} \alpha$ such that $a \alpha^{-1} \cap X^{\prime} \neq \emptyset$, let $x \in a \alpha^{-1} \cap$ $X^{\prime}$. Then $a \in \operatorname{Fix}\left(X_{x} \alpha\right)$, by (i), $\bar{a} \in \operatorname{Fix}\left(\left(X_{x} \varphi\right)(\alpha \varphi)\right)$. That is, $\bar{a} \in \operatorname{ran}(\alpha \varphi)$. Since $\bar{x}(\alpha \varphi)=\bar{a}\left(X_{x} \varphi\right)(\alpha \varphi)=\bar{a}$, it follows that $\bar{x} \in \bar{a}(\alpha \varphi)^{-1} \cap Y^{\prime}$. Then $\left(a \alpha^{-1} \cap X^{\prime}\right) \theta_{\varphi} \subseteq \bar{a}(\alpha \varphi)^{-1} \cap Y^{\prime}$. Similarly, by considering $\varphi^{-1}$ instead of $\varphi, \bar{a}(\alpha \varphi)^{-1} \cap Y^{\prime} \subseteq\left(a \alpha^{-1} \cap X^{\prime}\right) \theta_{\varphi}$. Thus the equality is obtained.

Lemma 4. For each $\alpha \in T_{O P}\left(X, X^{\prime}\right)$, if $b \in \operatorname{ran} \alpha$ and $b \alpha^{-1} \cap$ $X^{\prime}=\emptyset$, then $\bar{b} \in \operatorname{ran}(\alpha \varphi)$.

Proof. Let $b \in \operatorname{ran} \alpha$ and $b \alpha^{-1} \cap X^{\prime}=\emptyset$. Assume that $b$ is neither maximum nor minimum in $X^{\prime}$. Choose $a, c \in X^{\prime}$ such that $a<b<c$ and let $\epsilon_{b}=\left(\begin{array}{ccc}(\leftarrow b) & b & (b \rightarrow) \\ a & b & c\end{array}\right)$. Then $\epsilon_{b}$ is an idempotent with $b\left(\epsilon_{b}\right)^{-1} \cap X^{\prime}=\{b\}$. By Lemma $3, \bar{b}\left(\epsilon_{b} \varphi\right)^{-1} \cap Y^{\prime}=$ $\{\bar{b}\}$. Suppose in the contrary that $\bar{b} \notin \operatorname{ran}(\alpha \varphi)$. Then we have $\bar{b} \notin \operatorname{ran}\left((\alpha \varphi)\left(\epsilon_{b} \varphi\right)\right)$. Since $\left|\operatorname{ran}(\alpha \varphi)\left(\epsilon_{b} \varphi\right)\right|$ is finite, this guarantees the existence of an idempotent $\mu$ in $T_{\mathrm{OP}}\left(Y, Y^{\prime}\right)$ with $\operatorname{ran} \mu=\operatorname{ran}(\alpha \varphi)\left(\epsilon_{b} \varphi\right)$. Then $\mu \varphi^{-1}$ is an idempotent in $T_{\mathrm{OP}}\left(X, X^{\prime}\right)$, by Lemma $3, b \notin \operatorname{ran}\left(\mu \varphi^{-1}\right)$. However,

$$
\alpha \epsilon_{b}\left(\mu \varphi^{-1}\right)=\left((\alpha \varphi)\left(\epsilon_{b} \varphi\right) \mu\right) \varphi^{-1}=\left((\alpha \varphi)\left(\epsilon_{b} \varphi\right)\right) \varphi^{-1}=\alpha \epsilon_{b},
$$

which is a contradiction. If $b$ is either maximum or minimum, it can be proved in the same way by defining $\epsilon_{b}$ as before and choosing $a=b$ if $b$ is minimum, and $c=b$ if $b$ is maximum.

By Lemmas 3 and 4, the following proposition is directly obtained.

Proposition 5. For each $\alpha \in T_{O P}\left(X, X^{\prime}\right)$, we have the following.

(i) $(\operatorname{ran} \alpha) \theta_{\varphi}=\operatorname{ran}(\alpha \varphi)$.

(ii) For any $a \in \operatorname{ran} \alpha, \bar{a}(\alpha \varphi)^{-1} \cap Y^{\prime}=\left(a \alpha^{-1} \cap X^{\prime}\right) \theta_{\varphi}$.

This proposition leads us to define an interesting equivalence relation on the semigroup of full transformations with restricted range.

Given a transformation $\alpha: X \rightarrow X^{\prime}$, the $\alpha$-structure is the partial graph $\Gamma_{\alpha}$ which its components will be placed in the same order as their related elements in the range. Here we define an equivalence relation $\mathscr{K}$ on $T\left(X, X^{\prime}\right)$ by

$\alpha \mathscr{K} \beta \quad$ iff $\alpha$-structure and $\beta$-structure are the same. 
Indeed, it is equivalent to $\left.\alpha\right|_{X^{\prime}}=\left.\beta\right|_{X^{\prime}}$ and $\operatorname{ran} \alpha=\operatorname{ran} \beta$. The $\mathscr{K}$-class containing $\alpha$ is denoted by $\mathscr{K}_{\alpha}$. It is very clear that when $X^{\prime}=X, T\left(X, X^{\prime}\right)$ is $\mathscr{K}$-trivial. By Proposition 5, we have that $\mathscr{K}_{\alpha}$ and $\left(\mathscr{K}_{\alpha}\right) \varphi=\mathscr{K}_{\alpha \varphi}$ have the same structure for all $\alpha \in T_{\mathrm{OP}}\left(X, X^{\prime}\right)$.

Next, we will construct an extension of $\theta_{\varphi}$ to be an orderisomorphism on the adjusted chains.

Lemma 6. Suppose that two classes $\left[k_{1}\right]$ and $\left[k_{2}\right]$ are the minimum and the maximum of $\mathscr{A}\left\{X, X^{\prime}\right\}$, respectively. Let $a, b \in$ $X^{\prime}$ be such that $a<b$, and $A \subseteq\left[k_{1}\right]$ and $B \subseteq\left[k_{2}\right]$ as a lowerconvex subset and an upper-convex subset of $\left[k_{1}\right]$ and $\left[k_{2}\right]$, respectively. Then

$$
\left(\omega_{A_{a}: b}\right) \varphi=\omega_{C_{\bar{a}}: \bar{b}}, \quad\left(\omega_{a: B_{b}}\right) \varphi=\omega_{\bar{a}: D_{\bar{b}}}
$$

for some lower-convex $C$ and upper-convex $D$ of the minimum and the maximum of $\left\{Y \backslash Y^{\prime}\right\}$, respectively.

Proposition 7. For each $[k] \in\left\{X \backslash X^{\prime}\right\}$, there is a corresponding $\left[t_{k}\right] \in\left\{Y \backslash Y^{\prime}\right\}$ such that the extended map of $\theta_{\varphi}$ from $X^{\prime} \cup\{[k]\}$ onto $Y^{\prime} \cup\left\{\left[t_{k}\right]\right\}$ is an order-isomorphism. Moreover, $|[k]|=\left|\left[t_{k}\right]\right|$.

Proof. Let $[k] \in\left\{X \backslash X^{\prime}\right\}$ be such that $a<[k]<b$ for some $a, b \in X^{\prime}$. We choose $\omega_{a: A_{a}: b}$ as an idempotent in $T_{\mathrm{OP}}\left(X, X^{\prime}\right)$ whose range is $\{a, b\}$. Since two partial graphs of transformations $\Gamma_{\omega_{a: A_{a}: b}}$ and $\Gamma_{\left(\omega_{a: A_{a}: b}\right) \varphi}$ have the same structure, by Proposition 5, it follows that $\left(\mathscr{K}_{\omega_{a: A_{a}: b}}\right) \varphi=\mathscr{K}_{\left(\omega_{a: A_{a}: b}\right) \varphi}$. Due to the structure of $\Gamma_{\omega_{a: A_{a}: b}}$, the cardinality of $\mathscr{K}_{\omega_{a: A_{a}: b}}$ is depending only on $[k]$. Indeed, $\left|\mathscr{K}_{\omega_{a: A_{a}: b}}\right|=|[k]|$. This implies the existence of $\left[t_{k}\right] \in\left\{Y \backslash Y^{\prime}\right\}$ with $\bar{a}<\left[t_{k}\right]<\bar{b}$ and $\left|\mathscr{K}_{\left(\omega_{a: A_{a}: b}\right) \varphi}\right|=$ $\left|\left[t_{k}\right]\right|$.

Suppose $[k]$ is maximum (or minimum) in $\mathscr{A}\left\{X, X^{\prime}\right\}$. For any $a, b \in X^{\prime}$ such that $a<b$, we consider $\omega_{a:[k]_{b}}$ (or $\left.\omega_{[k]_{a}: b}\right)$. By Lemma 6 and using the same argument, our proof is finished.

From Proposition 7, the union of all these extensions form an order-isomorphism, denoted by $\widehat{\theta_{\varphi}}$ (with respect to $\theta_{\varphi}$ ), from $\mathscr{A}\left\{X, X^{\prime}\right\}$ onto $\mathscr{A}\left\{Y, Y^{\prime}\right\}$ such that

$$
x \longmapsto \bar{x}, \quad[k] \longmapsto\left[t_{k}\right]
$$

for $x \in X^{\prime}$ and $[k] \in\left\{X \backslash X^{\prime}\right\}$. We notice that $\widehat{\theta_{\varphi}}$ is an order-structural isomorphism (as defined in [5]). This conclusion results in the isomorphism theorems between the two semigroups for an infinite discrete chain.

Theorem 8. Let $X$ and $Y$ be infinite discrete chains. Then $T_{O P}\left(X, X^{\prime}\right) \cong T_{O P}\left(Y, Y^{\prime}\right)$ if and only if there is an order-(anti)isomorphism $\theta: X \rightarrow Y$ such that $\left(X^{\prime}\right) \theta=Y^{\prime}$.

Nevertheless, the property that $|[k]|=\left|\left[t_{k}\right]\right|=\left|([k]) \widehat{\theta_{\varphi}}\right|$ is not sufficient to determine the isomorphism for an uncountable chain. As a result, we study more of homomorphism properties associated with a class of $\left\{X \backslash X^{\prime}\right\}$.
Lemma 9. Let $[k] \in\left\{X \backslash X^{\prime}\right\}$ be such that $a<b<[k]<c$ (or $a<[k]<b<c)$ for some $a, b, c \in X^{\prime}$. Then for each convex subset $A$ of $[k]$,

$$
\left(\omega_{a: A_{b}: c}\right) \varphi=\omega_{\bar{a}: B_{\bar{b}}: \bar{c}}
$$

for some convex subset B of $\left[t_{k}\right] \in\left\{Y \backslash Y^{\prime}\right\}$ with $\bar{a}<\bar{b}<\left[t_{k}\right]<\bar{c}$ (or $\bar{a}<\left[t_{k}\right]<\bar{b}<\bar{c}$ ).

Proof. By Proposition 5, it follows that $\operatorname{ran}\left(\left(\omega_{a: A_{b}: c}\right) \varphi\right)=$ $\{\bar{a}, \bar{b}, \bar{c}\}$. Since $\left(a \omega_{a: A_{b}: c}^{-1} \cup c \omega_{a: A_{b}: c}^{-1}\right) \cap X^{\prime}=X^{\prime}$, by Lemma 3, we have that $\left(\bar{a}\left(\left(\omega_{a: A_{b}: c}\right) \varphi\right)^{-1} \cup \bar{c}\left(\left(\omega_{a: A_{b}: c}\right) \varphi\right)^{-1}\right) \cap Y^{\prime}=Y^{\prime}$. As $\left(\left(\omega_{a: A_{b}: c}\right) \varphi\right)$ is order-preserving such that $\bar{b}$ is in its range, there exists the unique class in $\left\{Y \backslash Y^{\prime}\right\}$, namely $\left[t_{k}\right]$, containing a convex subset $\bar{b}\left(\left(\omega_{a: A_{b}: c}\right) \varphi\right)^{-1}$.

Proposition 10. Let $[k] \in\left\{X \backslash X^{\prime}\right\}$ be such that $a<b<[k]<$ $c$ (or $a<[k]<b<c$ ) for some $a, b, c \in X^{\prime}$. Then for each $x \in[k]$,

$$
\left(\omega_{a:\{x\}_{b}: c}\right) \varphi=\omega_{\bar{a}:\{y\}_{b}: \bar{c}}
$$

for some $y \in\left[t_{k}\right]$.

Proof. Let $f$ and $g$ stand for two idempotents in $T_{\mathrm{OP}}\left(X, X^{\prime}\right)$ such that $\operatorname{ran} f=\operatorname{ran} g=\{a, c\}$ with $\{b, c\} \subseteq c f^{-1}$ and $\{a, b\} \subseteq$ $a g^{-1}$. Let $B$ be a convex subset of $[k]$ such that $|B|>1$. By Lemma 9, we obtain that $\left(\omega_{a: B_{b}: c}\right) \varphi=\omega_{\bar{a}: M_{\bar{b}}: \bar{c}}$ for some convex subset $M$ of $\left[t_{k}\right]$. Suppose in the contrary that $M=\{y\}$. We choose $L$ and $U$ as two convex subsets of $B$ which form a partition of $B$, and $L$ is a lower bound of $U$. Since $\omega_{a: U_{b}: c} g=$ $\omega_{a: B_{b}: c} g$, it follows that

$$
\left(\omega_{a: U_{b}: c} \varphi\right)(g \varphi)=\left(\omega_{a: B_{b}: c} \varphi\right)(g \varphi)=\omega_{\bar{a}:\{y\}_{\bar{b}}: \bar{c}}(g \varphi) .
$$

Then $\bar{b}\left(\omega_{a: U_{b}: c} \varphi\right)^{-1}$ is an upper-convex subset of $(\leftarrow y]$. Since $\omega_{a: L_{b}: c} f=\omega_{a: B_{b}: c} f$, we have

$$
\left(\omega_{a: L_{b}: c} \varphi\right)(f \varphi)=\left(\omega_{a: B_{b}: c} \varphi\right)(f \varphi)=\omega_{\bar{a}:\{y\}_{b}: \bar{c}}(f \varphi) .
$$

Then $\bar{b}\left(\omega_{a: L_{b}: c} \varphi\right)^{-1}$ is a lower-convex subset of $[y \rightarrow)$. It can be seen that $\omega_{a: L_{b}: c} g=\omega_{a: U_{b}: c} f$. Then $\left(\omega_{a: L_{b}: c} \varphi\right)(g \varphi)=$ $\left(\omega_{a: U_{b}: c} \varphi\right)(f \varphi)$ which contradicts to

$$
\begin{aligned}
\bar{a} & =\bar{b}(g \varphi)=y\left(\omega_{a: L_{b}: c} \varphi\right)(g \varphi)=y\left(\omega_{a: U_{b}: c} \varphi\right)(f \varphi) \\
& =\bar{b}(f \varphi)=\bar{c} .
\end{aligned}
$$

Proposition 11. For $a, b, c \in X^{\prime}$ with $a<b<c$, the following statements hold.

(i) If $[k]=\max \mathscr{A}\left\{X \backslash X^{\prime}\right\}$, then, for $x \in[k]$,

$$
\left(\omega_{a:[x \rightarrow)_{c}}\right) \varphi=\omega_{\bar{a}:[y \rightarrow)_{\bar{c}}} \text { for some } y \in\left[t_{k}\right] \text {. }
$$




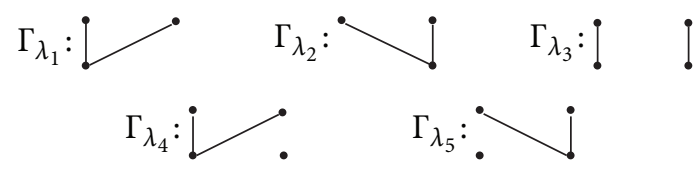

FIgURE 2

(ii) If $[k]=\min \mathscr{A}\left\{X \backslash X^{\prime}\right\}$, then, for $x \in[k]$,

$$
\left(\omega_{(\leftarrow x]_{a}: c}\right) \varphi=\omega_{(\leftarrow y]_{\bar{a}}: \bar{c}} \text { for some } y \in\left[t_{k}\right] .
$$

Proof. (i) Suppose $[k]=\max \mathscr{A}\left\{X \backslash X^{\prime}\right\}$. Let $x \in[k]$. Suppose that $(x \rightarrow) \neq \emptyset$. We let $\alpha=\left(\begin{array}{c}(\leftarrow x) x(x \rightarrow) \\ a b b\end{array}\right)$ and $\beta=(\underset{a}{(\leftarrow b)[b \rightarrow} \underset{c}{b})$. Clearly, $\alpha \beta=\omega_{a:[x \rightarrow)_{c}}$. Then $(\alpha \varphi)(\beta \varphi)=$ $\left(\omega_{a:[x \rightarrow)_{c}}\right) \varphi$. By applying the same process as in the proof of Proposition 10, we obtain that $\left|\bar{b}(\alpha \varphi)^{-1}\right|=1$. It is clear that $\bar{c}\left(\omega_{a:[x \rightarrow)_{c}} \varphi\right)^{-1}=\bar{b}(\alpha \varphi)^{-1} \dot{\cup} \bar{c}(\beta \varphi)^{-1}$ where $\bar{b}(\alpha \varphi)^{-1}$ is a lower-convex subset of $\bar{c}\left(\omega_{a:[x \rightarrow)_{c}} \varphi\right)^{-1}$. These imply that $\bar{c}\left(\omega_{a:[x \rightarrow)_{c}} \varphi\right)^{-1}=[y \rightarrow)$ for some $y \in\left[t_{k}\right]$.

(ii) can be proved similarly to (i).

\section{Isomorphism Theorems}

In the last section, we take care of the case $\left|X^{\prime}\right|=2$. For convenience, we here denote $T_{\mathrm{OP}}\left(X, X^{\prime}\right)$ by $\mathcal{O}\left[{ }_{M_{1}} 1_{M_{2}} 2_{M_{3}}\right]$ where $M_{1}, M_{2}$, and $M_{3}$ are three classes in $\left\{X \backslash X^{\prime}\right\}$. We observe that there are only 5 classes in $\mathcal{O}\left[{ }_{M_{1}} 1_{M_{2}} 2_{M_{3}}\right] / \mathscr{K}$ whose partial graph of transformations is one of the forms shown in Figure 2.

The following results are directly derived.

Lemma 12. For $\mathscr{K}_{\lambda_{i}} \in \mathcal{O}\left[{ }_{M_{1}} 1_{M_{2}} 2_{M_{3}}\right] / \mathscr{K},(i=1, \ldots, 5)$, we have that

(i) $\mathscr{K}_{\lambda_{1}}$ and $\mathscr{K}_{\lambda_{2}}$ are trivial,

(ii) $\left|\mathscr{K}_{\lambda_{3}}\right|=\left|M_{2}\right|+1$,

(iii) $\left|\mathscr{K}_{\lambda_{4}}\right|=\left|M_{3}\right|$ and $\left|\mathscr{K}_{\lambda_{5}}\right|=\left|M_{1}\right|$.

Proof. Since there are only two constant maps, (i) is proved. To show (ii), it is easy to see that each element in $M_{2}$ determines the consequent map in $\mathscr{K}_{\lambda_{3}}$ and vice versa. Hence the bijection between the two sets is constructed. The same idea can also be applied to show $\left|\mathscr{K}_{\lambda_{4}}\right|=\left|M_{3}\right|$ and $\left|\mathscr{K}_{\lambda_{5}}\right|=$ $\left|M_{1}\right|$.

Theorem 13. $\mathcal{O}\left[{ }_{M_{1}} 1_{M_{2}} 2_{M_{3}}\right] \cong \mathcal{O}\left[{ }_{N_{1}} \overline{1}_{N_{2}} \overline{2}_{N_{3}}\right]$ if and only if $\left|M_{i}\right|=\left|N_{i}\right|$ for all $i=1,2,3$.

Proof. Suppose that, for $i=1, \ldots, 5, \lambda_{i}$ and $\gamma_{i}$ are two representations of order-preserving maps having the same partial graph in $\mathcal{O}\left[M_{1} 1_{M_{2}} 2_{M_{3}}\right]$ and $\mathcal{O}\left[{ }_{N_{1}} \overline{1}_{N_{2}} \overline{2}_{N_{3}}\right]$, respectively. By Lemma 12, we let $f_{i}$ be a bijection from $\mathscr{K}_{\lambda_{i}}$ onto $\mathscr{K}_{\gamma_{i}}$ for $i=1, \ldots, 5$. To show that $\varphi:=f_{1} \cup f_{2} \cup \cdots \cup f_{5}$ : $\mathcal{O}\left[M_{1} 1_{M_{2}} 2_{M_{3}}\right] \rightarrow \mathcal{O}\left[{ }_{N_{1}} \overline{1}_{N_{2}} \overline{2}_{N_{3}}\right]$ is an isomorphism, we let $\alpha \in$ $\mathcal{O}\left[M_{1} 1_{M_{2}} 2_{M_{3}}\right]$. It is easy to see that the pairwise composition of five graph structures can be one of the following maps: for $\beta \in \mathcal{O}\left[{ }_{M_{1}} 1_{M_{2}} 2_{M_{3}}\right]$, either $\alpha \beta=\lambda_{1}, \alpha \beta=\lambda_{2}$ or $\alpha \beta=\alpha$ :

$$
\begin{array}{l|lllll} 
& \lambda_{1} & \lambda_{2} & \lambda_{3} & \lambda_{4} & \lambda_{5} \\
\hline \lambda_{1} & \lambda_{1} & \lambda_{2} & \lambda_{1} & \lambda_{1} & \lambda_{2} \\
\lambda_{2} & \lambda_{1} & \lambda_{2} & \lambda_{2} & \lambda_{1} & \lambda_{2} \\
\lambda_{3} & \lambda_{1} & \lambda_{2} & \lambda_{3} & \lambda_{1} & \lambda_{2} \\
\lambda_{4} & \lambda_{1} & \lambda_{2} & \lambda_{4} & \lambda_{1} & \lambda_{2} \\
\lambda_{5} & \lambda_{1} & \lambda_{2} & \lambda_{5} & \lambda_{1} & \lambda_{2}
\end{array}
$$

Suppose $\alpha \beta=\lambda_{1}$. One of the following statements hold:

(i) $\beta=\lambda_{1}$,

(ii) $\beta \in \mathscr{K}_{\lambda_{4}}$,

(iii) $\beta \in \mathscr{K}_{\lambda_{3}}$ and $\alpha=\lambda_{1}$.

It is clear that $(\alpha \varphi)(\beta \varphi)=\gamma_{1}=\left(\lambda_{1}\right) \varphi$.

For the rest, it can be proved directly.

Example 14. Let $X=\mathbb{R}, X^{\prime}=\{1,2\}, Y=[2,5)$, $Y^{\prime}=\{3,4\}$. Theorem 13 tells us that $\mathcal{O}\left[(-\infty, 1) 1_{(1,2)} 2_{(2, \infty)}\right] \cong$ $\mathcal{O}\left[{ }_{[2,3)} 3_{(3,4)} 4_{(4,5)}\right]$, yet it is clear that $\mathbb{R}$ and $[2,5)$ are not order or order-anti-isomorphic.

Next, we will prove that when $\left|X^{\prime}\right| \geq 3$, the converse of (2) holds.

Theorem 15. Suppose that $\left|X^{\prime}\right| \geq 3$. Then $T_{O P}\left(X, X^{\prime}\right) \cong$ $T_{O P}\left(Y, Y^{\prime}\right)$ if and only if there is an order-(anti)-isomorphism $\theta$ from $X$ onto $Y$ such that $\left(X^{\prime}\right) \theta=Y^{\prime}$.

Proof. It remains to show that, for each $[k] \in\left\{X \backslash X^{\prime}\right\},[k]$ and $\left[t_{k}\right]$ are order-isomorphic. Let $[k]$ be a class in $\left\{X \backslash X^{\prime}\right\}$. We will consider in two cases.

Case 1. Consider $[k]=\max \mathscr{A}\left\{X \backslash X^{\prime}\right\}$ or $\min \mathscr{A}\left\{X \backslash X^{\prime}\right\}$.

Without loss of generality we can assume that $[k]=$ $\max \mathscr{A}\left\{X \backslash X^{\prime}\right\}$. We choose $a, b, c \in X^{\prime}$ with $a<b<c$. For any $x, x^{\prime} \in[k]$ with $x<x^{\prime}$. Consider $\omega_{a:[x \rightarrow)_{c}}$ and $\omega_{a:\left[x^{\prime} \rightarrow\right)_{c}}$. By Proposition 11, we have $\left(\omega_{a:[x \rightarrow)_{c}}\right) \varphi=\omega_{\bar{a}:[y \rightarrow)_{\bar{c}}}$

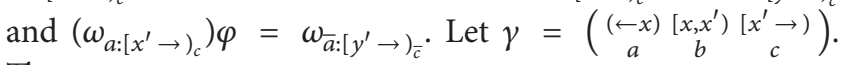
Then

$$
\begin{aligned}
& \gamma\left(\begin{array}{cc}
(\leftarrow b) & {[b \rightarrow)} \\
a & c
\end{array}\right)=\omega_{a:[x \rightarrow)_{c}} \\
& \gamma\left(\begin{array}{cc}
(\leftarrow b] & (b \rightarrow) \\
a & c
\end{array}\right)=\omega_{a:\left[x^{\prime} \rightarrow\right)_{c}} .
\end{aligned}
$$

It follows that

$$
\begin{aligned}
& (\gamma \varphi)\left(\begin{array}{cc}
(\leftarrow b) & {[b \rightarrow)} \\
a & c
\end{array}\right) \varphi=\left(\omega_{a:[x \rightarrow)_{c}}\right) \varphi=\omega_{\bar{a}:[y \rightarrow)_{\bar{c}}}, \\
& (\gamma \varphi)\left(\begin{array}{cc}
(\leftarrow b] & (b \rightarrow) \\
a & c
\end{array}\right) \varphi=\left(\omega_{a:\left[x^{\prime} \rightarrow\right)_{c}}\right) \varphi=\omega_{\bar{a}:\left[y^{\prime} \rightarrow\right)_{\bar{c}} \cdot}
\end{aligned}
$$


Since $\left[\begin{array}{l}y\end{array}\right)=\bar{c}\left(\omega_{\bar{a}:[y \rightarrow)_{\bar{c}}}\right)^{-1}=\bar{b}(\gamma \varphi)^{-1} \dot{\cup} \bar{c}(\gamma \varphi)^{-1}$ and $\left[y^{\prime} \rightarrow\right)=\bar{c}\left(\omega_{\bar{a}:\left[y^{\prime} \rightarrow\right)_{\bar{c}}}\right)^{-1}=\bar{c}(\gamma \varphi)^{-1}$, these imply that $y<y^{\prime}$.

Case 2. $[k]$ is neither $\max \mathscr{A}\left\{X \backslash X^{\prime}\right\}$ nor $\min \mathscr{A}\left\{X \backslash X^{\prime}\right\}$.

Then there are $a, b, c \in X^{\prime}$ such that $a<b<[k]<c$ or $a<[k]<b<c$.

By using Proposition 10 and following the same proof as in Case 1, we derive the result.

\section{Conflict of Interests}

The authors declare that there is no conflict of interests regarding the publication of this paper.

\section{Acknowledgments}

This research was partially supported by SWU endowment fund year 2014 and also by WU. The authors thank the (anonymous) referees for helpful comments.

\section{References}

[1] J. M. Howie, "Products of idempotents in certain semigroups of transformations," Proceedings of the Edinburgh Mathematical Society, vol. 17, no. 3, pp. 223-236, 1971.

[2] J. S. Symons, "Some results concerning a transformation semigroup," Australian Mathematical Society, vol. 19, no. 4, pp. 413425, 1975.

[3] A. Umar, "Semigroups of order-decreasing transformations: the isomorphism theorem," Semigroup Forum, vol. 53, no. 2, pp. 220-224, 1996.

[4] T. Saitô, K. Aoki, and K. Kajitori, "Remarks on isomorphisms of regressive transformation semigroups," Semigroup Forum, vol. 53, no. 1, pp. 129-134, 1996.

[5] P. Udomkavanich and P. Jitjankarn, "A classification of regressive transformation semigroups on chains," Semigroup Forum, vol. 85, no. 3, pp. 559-570, 2012.

[6] E. S. Lyapin, Semigroups, American Mathematical Society, Providence, RI, USA, 1974.

[7] Y. Kemprasit, W. Mora, and T. Rungratgasame, "Isomorphism theorems for semigroups of order-preserving partial transformations," International Journal of Algebra, vol. 4, no. 17-20, pp. 799-808, 2010.

[8] V. H. Fernandes, P. Honyam, T. M. Quinteiro, and B. Singha, "On semigroups of endomorphisms of a chain with restricted range," Semigroup Forum, vol. 89, no. 1, pp. 77-104, 2014. 


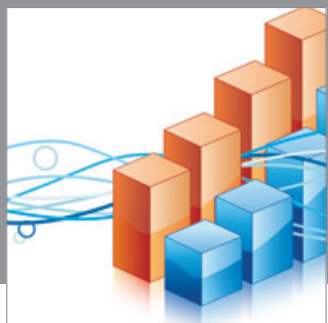

Advances in

Operations Research

mansans

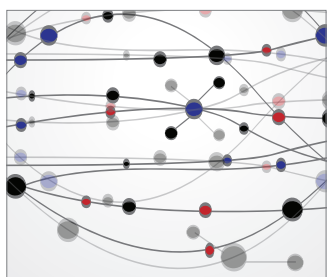

The Scientific World Journal
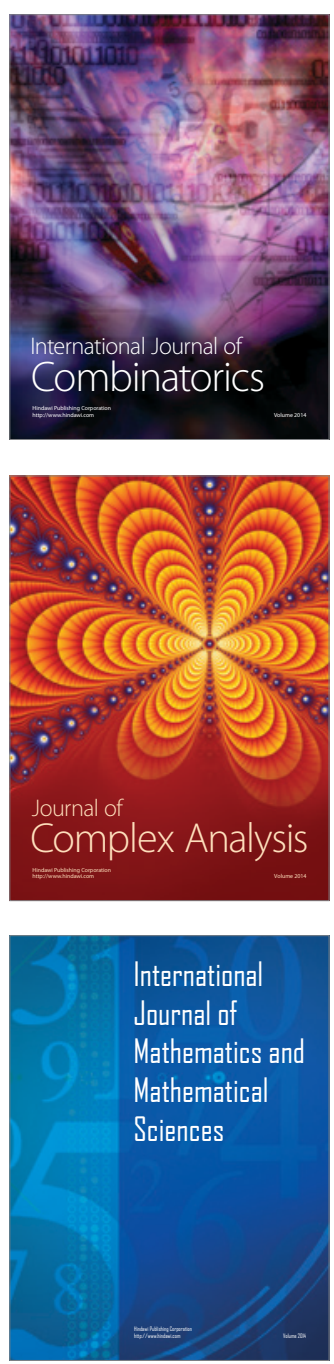
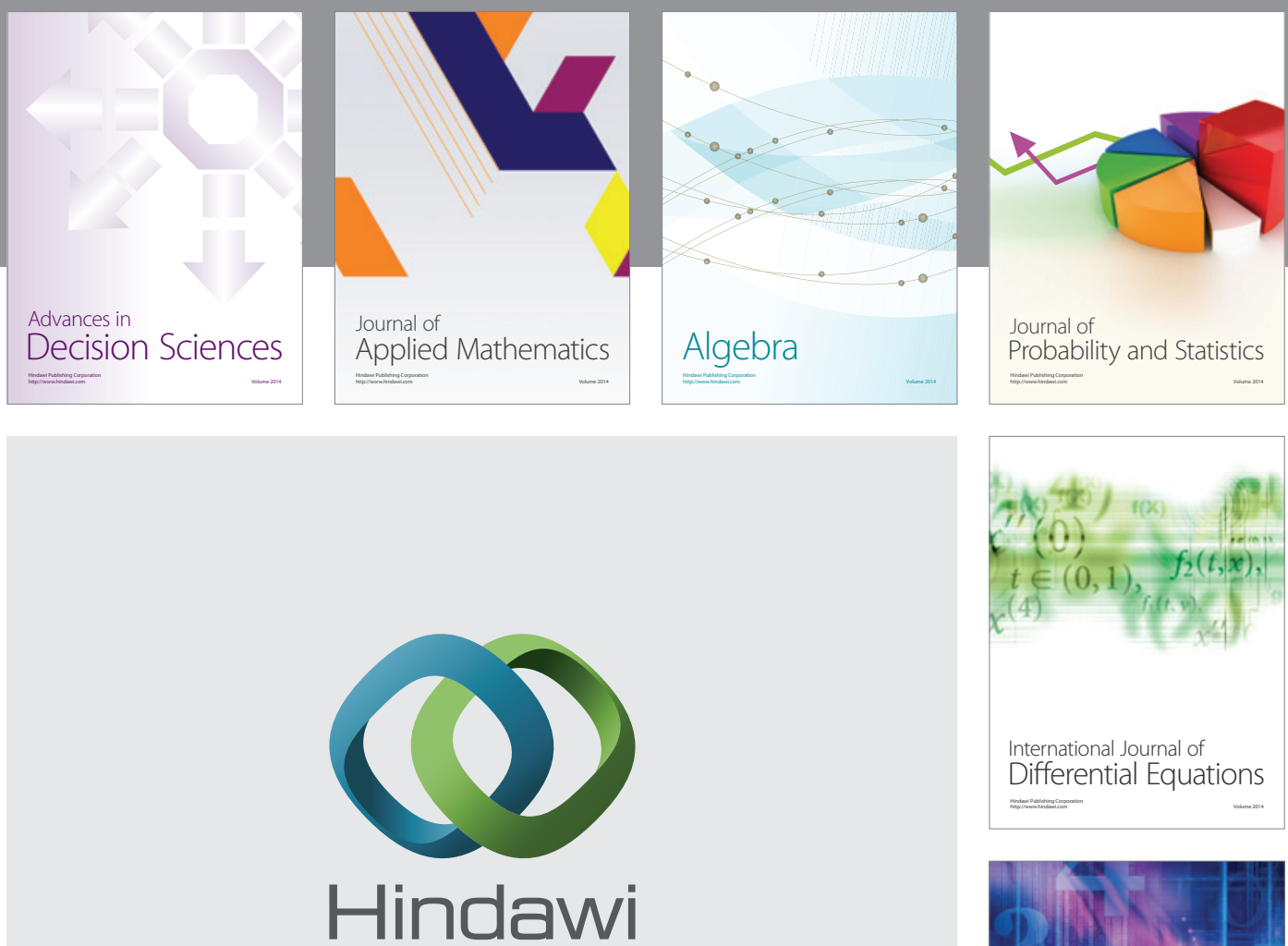

Submit your manuscripts at http://www.hindawi.com
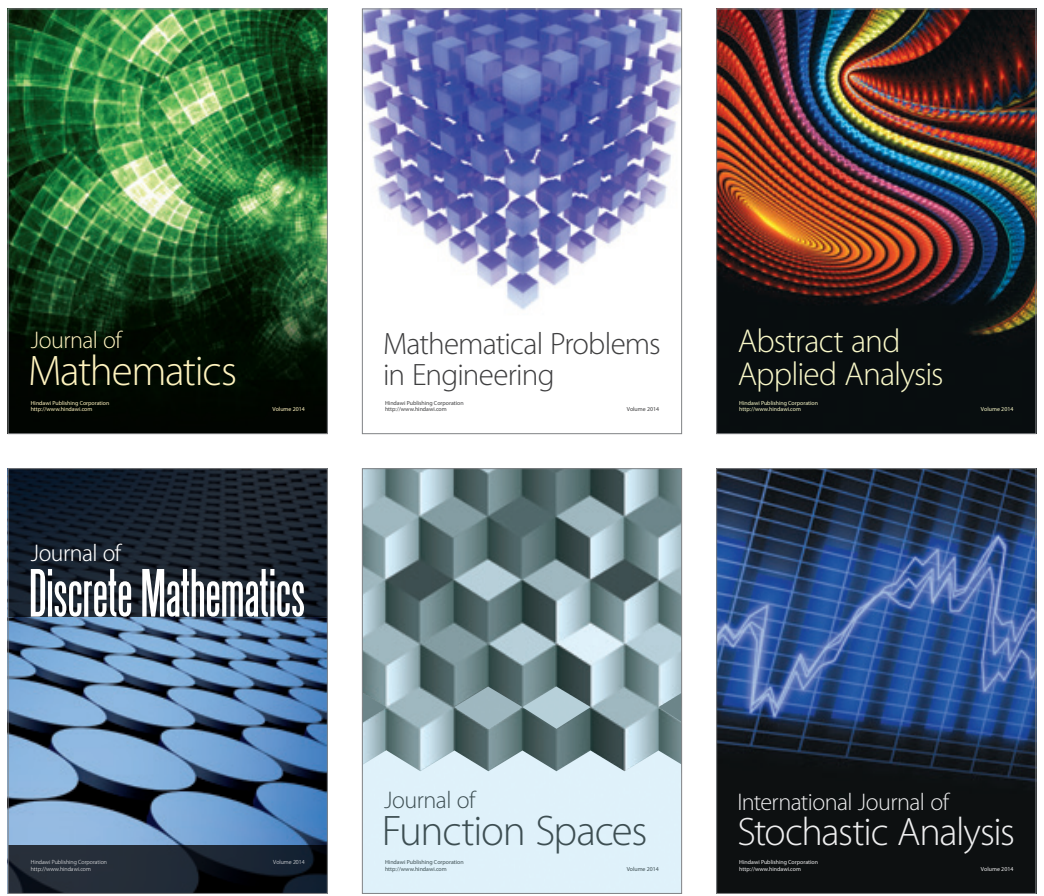

Journal of

Function Spaces

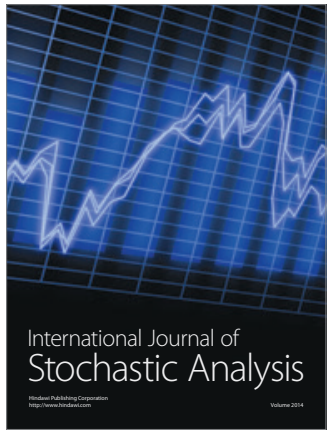

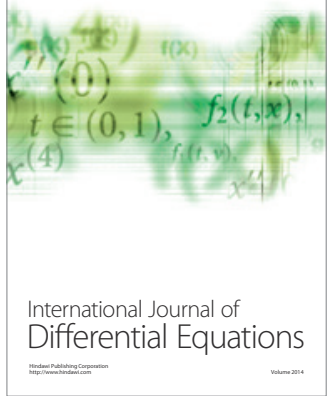
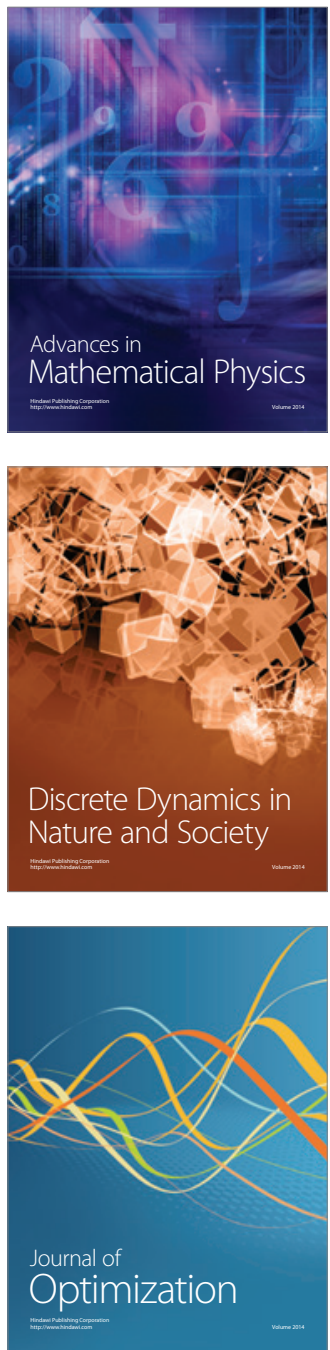